\title{
COBERTURA DO EXAME PREVENTIVO DE CÂNCER DE COLO DE ÚTERO EM UMA UNIDADE DE SAÚDE DA FAMÍLIA
}

Maria Auxiliadora de Souza Gerk1, Mirian Yuriko Girata², Cristina Brandt Nunes³, Sandra Luzinete Félix de Freitas ${ }^{4}$

${ }^{1}$ Enfermeira. Doutora em Ciências. Universidade Federal do Mato Grosso do Sul. Campo Grande-MS-Brasil.

²Enfermeira. Especialista em Gerência de Unidade Básica de Saúde. Universidade Federal do Mato Grosso do Sul. Campo Grande-MS-Brasil. ${ }^{3}$ Enfermeira. Doutora em Ciências. Universidade Federal do Mato Grosso do Sul. Campo Grande-MS-Brasil.

${ }^{4}$ Enfermeira.MestreemSaúdeeDesenvolvimentoda RegiãoCentro-Oeste. UniversidadeFederal doMatoGrossodoSul.CampoGrande-MS-Brasil.

RESUMO: Este estudo quantitativo estimou retrospectivamente a cobertura do exame de Papanicolaou na área adscrita à Unidade de Saúde da Família Santa Rita, no município de Três Lagoas, MS, utilizando dados disponíveis em sistemas de informação municipais e federais e em prontuários eletrônicos referentes a 361 mulheres residentes nessa área. A cobertura do exame foi de 82,54\% em mulheres de 20 a 59 anos de idade. Não foram constatadas alterações celulares em $94,7 \%$ das amostras. Além de revelar o quadro epidemiológico do câncer cérvico-uterino, o estudo traz subsídios para aprimorar as estratégias em curso e aperfeiçoar as atividades em saúde da mulher.

DESCRITORES: Neoplasias do colo do útero; Prevenção primária; Saúde da mulher; Enfermagem em saúde comunitária.

\section{COVERAGE OF THE PREVENTIVE TEST FOR CERVICAL CANCER IN A FAMILY HEALTH CENTER}

\begin{abstract}
This quantitative study retrospectively estimated the coverage of the Papanicolaou test in the area covered by the Santa Rita Family Health Center, in the city of Três Lagoas, in the State of Mato Grosso do Sul (MS). It used data available in Municipal and Federal Information Systems, and in electronic hospital records referent to 361 women resident in this area. The coverage of the test was $82.54 \%$ among women aged between 20 and 59 years old. Cellular changes were not observed in $94.7 \%$ of the samples. In addition to showing the epidemiological situation for cervical uterine cancer, the study provides support for improving strategies currently in use and for improving activities in women's health.
\end{abstract}

DESCRIPTORS: Cervical cancers; Primary prevention; Women's health; Nursing in community health.

\section{COBERTURA DEL EXAMEN PREVENTIVO DE CÁNCER DE CUELLO DE ÚTERO EN UNA UNIDAD DE SALUD DE LA FAMILIA}

RESUMEN: Este estudio cuantitativo hizo la estimativa retrospectiva de la cobertura del examen de Papanicolaou en el área adscrita a la Unidad de Salud de la Familia Santa Rita, en el municipio de Três Lagoas, MS, utilizando datos disponibles en sistemas de información municipales y federales y en prontuarios electrónicos referentes a 361 mujeres que viven en ese área. La cobertura del examen fue de $82,54 \%$ en mujeres de 20 a 59 años de edad. No fueron constatadas alteraciones celulares en $94,7 \%$ de las muestras. Además de revelar el cuadro epidemiológico del cáncer cervical uterino, el estudio trae subsidios para perfeccionar estrategias en curso y mejorar las actividades en salud de la mujer.

DESCRIPTORES: Neoplasias del cuello del útero; Prevención primaria; Salud de la mujer; Enfermería en salud comunitaria. 


\section{INTRODUÇÃO}

Conforme relatório da Organização Mundial da Saúde (OMS), dos 57 milhões de óbitos mundiais em 2008, 36 milhões, ou seja, 63\% foram decorrentes de doenças não-transmissíveis, dentre elas o câncer ${ }^{(1)}$.

Nas Américas, cerca de 80.710 mulheres são anualmente diagnosticadas com câncer de colo de útero e, dentre estas, 36.100 morrem em consequência deste tipo de câncer. Estimase para 2030 um aumento de $70 \%$ de novos casos de câncer de colo de útero e de mama na América Latina e Caribe ${ }^{(2)}$. No Brasil, previram-se 518.510 novos casos de câncer para o período 2012-2013 ${ }^{(3)}$.

Atualmente, no Brasil, os cânceres de maior incidência entre mulheres são os de pele não melanoma, de mama, de colo de útero, de cólon e reto e de glândula tireoide ${ }^{(3)}$. Em países menos desenvolvidos, o câncer de colo de útero tem incidência maior que em nações mais desenvolvidas, sendo evidenciado já na faixa etária dos 20 aos 29 anos, embora seu risco aumente dos 45 aos $49 \operatorname{anos}^{(4)}$.

O câncer de colo de útero é o segundo tipo mais frequente de câncer nas regiões CentroOeste e Nordeste do Brasil, ocorrendo em 28 a cada 100 mil mulheres e em 18 a cada 100 mil, respectivamente ${ }^{(3)}$.

No Mato Grosso do Sul, sua incidência em 2001 foi de 14,68 casos por 100 mil mulheres, com estimativa de 35,13 por 100 mil para $2013^{(5)}$. Tal aumento pode ser associado à melhoria no diagnóstico, a um maior acesso aos serviços de saúde ou à maior exposição da população a fatores de risco para câncer de colo de útero ${ }^{(5)}$.

Essa forma da doença é a que apresenta maior potencial de prevenção e cura quando diagnosticada precocemente ${ }^{(4)}$. O exame citopatológico cérvico-uterino, também denominado preventivo de câncer de colo de útero ou exame de Papanicolaou, é um método simples que permite detectar alterações da cérvice uterina a partir de células descamadas do epitélio e constitui até hoje o método diagnóstico mais indicado, por ser rápido, indolor, de fácil execução e de baixo custo(6).

É também a estratégia de rastreamento recomendada pelo Ministério da Saúde prioritariamente para mulheres de 25 a 64 anos. Nessa faixa etária, a repetição do exame deverá ser realizada a cada três anos após dois exames em anos consecutivos com resultados negativos. Após essa idade, o exame poderá ser interrompido se a mulher apresentar dois exames negativos consecutivos nos últimos cinco anos. Mulheres com mais de 64 anos que nunca o efetuaram deverão realizar dois preventivos com intervalo de um a três anos. Se ambos forem negativos, poderão ser dispensadas de exames adicionais $^{(7-8)}$.

O exame preventivo faz parte da Atenção Básica de Saúde, que abrange ações voltadas à promoção e proteção da saúde, à prevenção de agravos, ao diagnóstico, ao tratamento, à reabilitação e à manutenção da saúde. A Atenção Básica é também a porta de entrada da maioria dos usuários no sistema de saúde e "representa o acúmulo e o aperfeiçoamento da política de saúde" para efetiva consolidação do SUS "como política pública voltada para a garantia de direitos constitucionais de cidadania"(9:25).

O presente estudo teve como objetivo estimar a cobertura do exame citopatológico do câncer de colo uterino em mulheres da área adscrita à Unidade de Saúde da Família (USF) Santa Rita no município sul-mato-grossense de Três Lagoas.

\section{MÉTODO}

Para esta pesquisa quantitativa foram retrospectivamente consultados dados disponíveis em prontuários eletrônicos e em livro de registros de exames da USF Santa Rita, no município de Três Lagoas-MS e, informações armazenadas nos seguintes bancos de dados: Sistema de Informação do Câncer de Colo Uterino (Siscolo), Sistema de Informação da Atenção Básica (SIAB) e CONSULFARMA (sistema de informação utilizado pelo município).

Foram pesquisados os registros referentes a mulheres residentes na área adscrita à USF Santa Rita que haviam realizado exame citopatológico cérvico-uterino de janeiro a dezembro de 2011. Para a coleta dos dados dos prontuários, elaborou-se um instrumento focalizando as seguintes variáveis: idade (em anos) das mulheres que realizaram o exame preventivo em 2011 e 
resultados dos exames coletados (incluindo flora microbiana e identificação de alteração de lesões precursoras).

A pesquisa foi empreendida após aprovação pelo Comitê de Ética em Pesquisa em Seres Humanos da Universidade Federal de Mato Grosso do Sul (parecer CAAE 01716012.3.0000.0021), em atendimento à Resolução 196/96 do Conselho Nacional de Saúde.

\section{RESULTADOS}

Entreas 361 mulheres que realizaram oexame na USF Santa Rita em 2011, predominaram os grupos etários de 20 a 39 anos (49,9\%) e de 40 a 49 (21\%).

O Gráfico 1 correlaciona o número de exames realizados, segundo faixa etária, e de mulheres cadastradas na unidade de saúde.

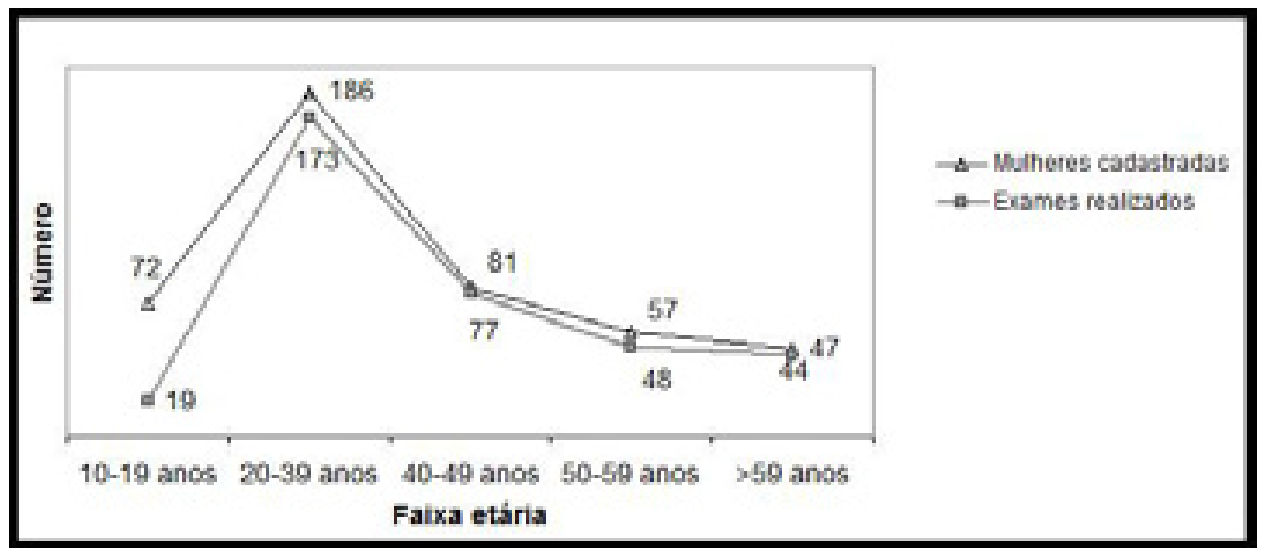

Gráfico 1 - Número de exames citopatológicos cérvico-uterinos e número de mulheres cadastradas na Unidade de Saúde da Família Santa Rita, segundo faixa etária. Três Lagoas-MS-Brasil, 2011

Todas as amostras de esfregaço foram consideradas satisfatórias para processamento pelo Laboratório de PatologiaMunicipal, queasanalisou. Dototal deamostras, 94,7\% (341) revelaram-se citologicamente normais.

Os micro-organismos identificados em $100 \%$ (361) das amostras foram cocos (28\%), bacilos (21\%), lactobacilos ácidos (11\%), Gadnerella vaginalis (7\%), Candida albicans (4\%) e Trichomonas vaginalis (3\%).

Detectaram-se alterações citológicas em 18 mulheres, $11(61,1 \%)$ das quais tinham de 20 a 39 anos. Observaram-se alterações escamosas atípicas de significado indeterminado possivelmente nãoneoplásicas (ASC-US) em 13 pacientes (72,2\%). Quatro $(22,2 \%)$ apresentaram lesão intraepitelial de baixo grau, compreendendo efeito citopático por HPV e neoplasia intraepitelial cervical (NIC) de grau I. Uma paciente realizou colposcopia e biópsia, com identificação de NIC de grau II. Houve um caso (5,5\%) de lesão intraepitelial de alto grau, compreendendo NIC de grau II e III, havendo-se procedido a colposcopia e biópsia com subsequente cirurgia de alta frequência.

A maior incidência $(61,1 \%)$ de alterações celulares (Gráfico 2) ocorreu em mulheres de 20 a 39 anos - faixa etária considerada pelo Instituto Nacional do Câncer $\left(\right.$ INCA) ${ }^{(7)}$ como a população-alvo por necessitar exame citopatológico periódico para o rastreamento de lesões precursoras de câncer de colo de útero.

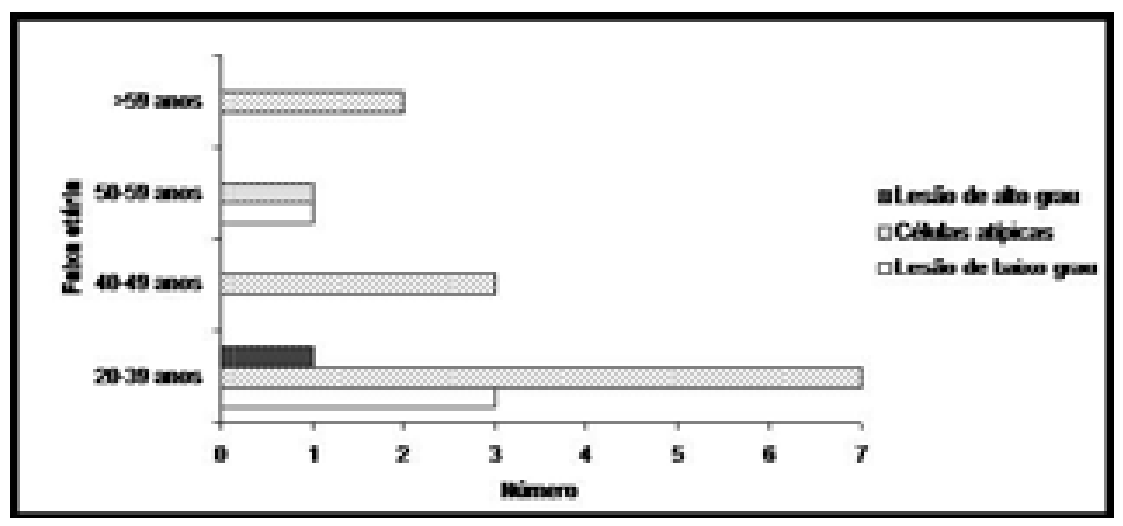

Gráfico 2 - Exames citopatológicos cérvico-uterinos alterados (n=18), por faixa etária, na Unidade de Saúde da Família Santa Rita. Três Lagoas-MS-Brasil, 2011 


\section{DISCUSSÃO}

Predominaram (82,54\%, ou 298) exames na faixa etária de 20 a 59 anos, corroborando achados de outras pesquisas ${ }^{(10-11)}$ sobre a população definida pelo INCA ${ }^{(7)}$ como alvo para detecção precoce de lesões precursoras de neoplasia (mulheres de 25 a 64 anos). Além disso, superou-se a cobertura de $80 \%$ preconizada pela $\mathrm{OMS}^{(8)}$. Estudos realizados em outros municípios revelaram coberturas de exame preventivo de $72,2 \%{ }^{(12)}, 66,3 \%{ }^{(13)}$ e $67 \%{ }^{(14)}$, aquém portanto do estipulado por essa entidade ${ }^{(15)}$.

Todos os esfregaços foram considerados, pelo Laboratório de Patologia Municipal, como satisfatórios para processamento e os resultados citológicos revelaram-se normais em 94,7\% das amostras. A adequação das amostras influencia o resultado do exame, uma vez que a presença de secreção purulenta ou de sangue, geralmente decorrente de coleta insatisfatória do material, prejudica a análise dos esfregaços citopatológicos ${ }^{(16)}$. Infere-se, portanto que a coleta dessas amostras tenha sido adequadamente realizada pelas enfermeiras atuantes na USF Santa Rita.

A análise microbiológica revelou predomínio de bactérias da microbiota vaginal, corroborando os achados de um estudo ${ }^{(17)}$ realizado no período de 2005 a 2009, em que 89,9\% (368) das amostras continham micro-organismos da microbiota vaginal. Achados semelhantes foram também encontrados em outra pesquisa ${ }^{(18)}$ realizada no período de março de 2008 a outubro de 2011, referentes a uma Unidade Básica de Saúde, que revelaram predomínio de cocos, bacilos e lactobacilos. No presente estudo, os cocos, bacilos e lactobacilos predominantes eram constituintes da microbiota vaginal, portanto não caracterizando infecção e não necessitando de tratamento caso a paciente esteja assintomática ${ }^{(7)}$.

A incidência de alterações celulares mostrou-se reduzida, perfazendo apenas $4,9 \%{ }^{(18)}$ das amostras analisadas, sendo que o diagnóstico de ASC-US predominou neste grupo, em concordância com os achados de outro estudo ${ }^{(19)}$. Outras pesquisas $^{(10-11,17)}$ apontaram baixa incidência de alterações celulares.

Dentre as 18 mulheres que apresentaram alterações celulares, 11 (61,1\%) encontravamse com idade entre 20 e 39 anos. Resultados semelhantes foram apontados em estudo realizado no Rio Grande do Norte ${ }^{(20)}$, no período de janeiro de 2000 a março de 2004, em que os resultados mais frequentes para NIC I, II e III apresentaram-se nessa mesma faixa etária.

As medidas educativas, seja por meio das consultas de Enfermagem ou das rodas de conversa, são essenciais para a mudança de comportamento da população, ou seja, para o desenvolvimento de atitudes preventivas e de promoção da saúde ${ }^{(21)}$.

A equipe de saúde, em especial o enfermeiro, que demonstra sensibilização às necessidades da clientela e que, com ela estabelece vínculo, aumenta a probabilidade de adesão à realização do exame preventivo do câncer do colo do útero $^{(22)}$.

\section{CONCLUSÃO}

Todas as amostras foram consideradas satisfatórias para processamento. Predominou a presença de micro-organismos da microbiota vaginal. Em apenas um caso constatou-se alteração intraepitelial de alto grau; em 13 mulheres foram encontradas ASC-US e quatro apresentaram lesão intraepitelial de baixo grau, compreendendo efeito citopático por HPV e NIC de grau I.

Dentre as 18 mulheres que apresentaram alterações celulares, 11 (61,1\%) encontravam-se com idade entre 20 e 39 anos, ou seja, no período de vida reprodutivo.

Apesar de este estudo ter evidenciado uma cobertura e coletas satisfatórias do exame preventivo do câncer do colo do útero, há necessidade de incremento nas estratégias desenvolvidas na USF Santa Rita para a sua prevenção, como as visitas domiciliares às mulheres que não retornam para buscar o resultado do exame ou que o realizam com um intervalo acima de três anos, agendamento das consultas de enfermagem que possibilitam um momento privilegiado para educação individual em saúde e formação de vínculo, criação de rodas de conversa para esclarecimentos das dúvidas e troca de experiência entre as mulheres.

\section{REFERÊNCIAS}

1. World Health Organization (WHO). Global Health Observatory. Deaths from NCDs. WHO, [Internet] 2008 [acessoem20out2011]. Disponível: http://www.who.int/gho/ ncd/mortality_morbidity/ncd_total_text/en/index.html. 
2. Organización Panamericana de la Salud (OPAS). Nueva iniciativa multisectorial buscará prevenir casos nuevos y muertes por cáncer cervicouterino y de mamas en las Américas. OPAS, [Internet] 2013 [acesso em 15 abr 2013]. Disponível: http://new.paho.org/hq/index

3. INCA. Instituto nacional do Câncer José Alencar Gomes da Silva. Coordenação Geral de Ações Estratégicas. Coordenação de Prevenção e Vigilância. Estimativa 2012: incidência de câncer no Brasil. Rio de Janeiro: INCA; 2011.

4. INCA. Instituto nacional do Câncer. Estimativa 2010: incidência de câncer no Brasil. INCA, [Internet] 2009 [acesso em 15 abr 2013]. Disponível: http://www2.inca. gov.br/wps/wcm/connect/agencianoticias/site/home/ noticias/2009/lancamento_estimativa_2010

5. Freitas HG, Silva MA, Thuler LCS. Câncer do colo do útero no estado de Mato Grosso do Sul: detecção precoce, incidência e mortalidade. Rev. bras. cancerol. [Internet] 2012;58(3) [acesso em 01 jul 2013]. Disponível: http:// www.inca.gov.br/rbc/n_58/v03/pdf/09_artigo_cancer_ colo_utero_estado_mato_grosso_sul_deteccao_precoce_ incidencia_mortalidade.pdf

6. Fernandes JV, Rodrigues SHL, Costa YGAS, Silva LCM, Brito AML, et al. Conhecimentos, atitudes e prática do exame de Papanicolaou por mulheres, Nordeste do Brasil. Rev. Saúde Públ. [Internet] 2009;43(5) [acesso em 24 jun 2011]. Disponível: http://dx.doi.org/10.1590/S003489102009005000055

7. INCA. Instituto Nacional do Câncer. Coordenação Geral de Ações Estratégicas. Divisão de Apoio à Rede de Atenção Oncológica. Diretrizes brasileiras para o rastreamento do câncer do colo do útero. Rio de Janeiro: INCA [Internet] 2011 [acesso em 5 jul 2011]. Disponível: http://www2.inca.gov.br/wps/wcm/ connect/agencianoticias/site/home/noticias/2011/ novas_diretrizes_para_rastreamento_do_cancer_ do_colo_do_utero_no_brasil_serao_lancadas_nesta_ segunda

8. World Health Organization (WHO). National cancer control programmes: policies and managerial guidelines. $2^{\text {a }}$ ed. Geneva: WHO [Internet] 2008 [acesso em 01 jul de 2013]. Disponível:http://www.who.int/reproductivehealth/ publications/cancers/9241545577/en/index.html

9. Ministério da Saúde (BR). Secretaria de Atenção à Saúde. Departamento de Atenção Básica. Cadernos de atenção básica: controle dos cânceres do colo do útero e da mama. $2^{a}$ ed. Brasília: Ministério da Saúde; 2013.

10. Melo SCCS, Prates L, Carvalho MDB, Marcon SS, Pelloso SM. Alterações citopatológicas e fatores de risco para a ocorrência do câncer de colo uterino. Rev. Gaúcha Enferm. [Internet] 2009;30(4) [acesso em 1 ago 2012]. Disponível: http://seer.ufrgs.br/RevistaGauchadeEnfermagem/article/ download/8959/7535

11. Risso VBS, Soares MC. Avaliação dos registros do exame citopatológico em uma unidade básica de saúde em Rio Grande-RS. Rev. enferm. saúde. [Internet] 2011;1(1) [acesso em 16 abr 2012]. Disponível: http://periodicos.ufpel.edu. br/ojs2/index.php/enfermagem/article/viewFile/3410/2801

12. Costa JSD, Olinto MTA, Gigante DP, Menezes AMB, Macedo $S$, et al. Cobertura do exame citopatológico na cidade de Pelotas, Rio Grande do Sul, Brasil. Cad. Saúde Pública. [Internet] 2003;19(1):191-7 [acesso em 14 abr 2012]. Disponível: http://dx.doi.org/10.1590/S0102311X2003000100021

13. Gonçalves CV, Sassi RM, Oliveira Netto I, Castro NB, Bortolomedi AP. Cobertura do citopatológico do colo uterino em Unidades Básicas de Saúde da Família. Rev. Bras. Ginecol. Obstet. [Internet] 2011;33(9) [acesso em 10 abr 2012]. Disponível: http://dx.doi.org/10.1590/S010072032011000900007

14. Albuquerque KM, Frias PG, Andrade CLT, Aquino EML, Menezes G, et al. Cobertura do teste de Papanicolaou e fatores associados à não-realização: um olhar sobre o Programa de Prevenção do Câncer do Colo do Útero em Pernambuco, Brasil. Cad. Saúde Pública. [Internet] 2009;25(2) [acesso em 20 Jul 2012]. Disponível: http:// dx.doi.org/10.1590/S0102-311X2009001400012

15. World Health Organization (WHO). Manual on the prevention and control of common cancers. Geneve: WHO; 1998.

16. Amaral RG, Manrique EJC, Guimarães JV, Souza PJ, Mignoli $J R Q$, et al. Influência da adequabilidade da amostra sobre a detecção das lesões precursoras do câncer cervical. Rev. Bras. Ginecol. Obstet. [Internet] 2008;30(11) [acesso em 4 fev 2012]. Disponível: http://dx.doi.org/10.1590/S010072032008001100005

17. Silva PV, Araujo A, Araujo MRN. Análise da cobertura do exame citopatológico do colo do útero no município de Doresópolis-MG. R. Enferm. Cent. O. Min. [Internet] 2011;1(2) [acesso em 15 ago 2012]. Disponível: http://www. seer.ufsj.edu.br/index.php/recom/article/download/41/133

18. Bringuel APV, Rodrigues MPF, Vidal ECF. Análise dos laudos de Papanicolaou realizados em uma Unidade Básica de Saúde. Cogitare enferm. [Internet] 2012;17(4) [acesso em 01 jul 2013]. Disponível: http://connection.ebscohost.com/c/ articles/87634082/an-lise-dos-laudos-de-papanicolaourealizados-em-uma-unidade-b-sica-de-sa-de

19. Fernandes F, Furtado $Y$, Russomano F, Silva KS, Silveira $\mathrm{R}$, et al. Diagnóstico citopatológico de ASC-US e ASC-H no Serviço Integrado Tecnológico em Citologia do INCA. Rev. bras. cancerol. [Internet] 2012;58(3) [acesso em 01 jul 2013]. Disponível: http://www.inca.gov.br/rbc//n_58/v03/ pdf/15_artigo_diagnostico_citopatologico_asc_us_asc_h_ servico_integrado_tecnologico_citologia_inca.pdf

20. Medeiros VCRD, Medeiros RC, Moraes LM, Menezes Filho JB, Ramos ESN, et al. Câncer de Colo de Útero: Análise Epidemiológica e Citopatológica no Estado do Rio Grande 
do Norte. RBAC [Internet] 2005;33(9):227-31 [acesso em 16 dez 2013]. Disponível: http://bases.bireme.br/cgibin/ wxislind.exe/iah/online/?lsisScript=iah/iah.

21. Ferreira MLSM. Motivos que influenciam a não realização do exame de Papanicolaou segundo a percepção de mulheres. Esc. Anna Nery. [Internet] 2009;13(2):37884 [acesso em 17 dez 2013]. Disponível: http://dx.doi. org/10.1590/S1414-81452009000200020

22. Moura ADA, Silva SMG, Farias LM, Ferroza AR. Conhecimento e Motivações das mulheres acerca do exame de Papanicolaou: subsídios para a prática de Enfermagem. Rev. Rene. [Internet] 2010;11(1):94-104 [acesso em 16 dez 2013]. Disponível: http://www. repositorio.ufc.br/bitstream/riufc/4013/1/2010_art_ adamoura.pdf 